vectrode through the brain. In the most common, 'blind' version, researchers do this without being able to see the neurons. The scientist must continually apply pressure to push brain matter away from the pipette, but when a rise in electrode resistance indicates that a cell is nearby, they must switch to suction at just the right moment to seal a tiny patch of the neuron's membrane against the pipette's super-thin tip. With an additional burst of suction, the researcher can then make a tiny hole in the cell membrane to record the neuron's activity. Hitting the neuron at the wrong angle, misregulating the pressure and numerous other variables often derail recordings.

"Every step has a certain failure rate, and these multiply throughout the process," says Boyden. Experienced practitioners report success rates of between $20 \%$ and $60 \%$.

Boyden and Forest decided to automate this tricky technique. Their robot does not outperform human experts yet, but its average success rate is around $33 \%$ in tests on mice. The device, which runs on the commercial programming platform LabVIEW, only requires researchers to position the animal and the pipette. A computer algorithm then controls the pipette's internal pressure and its progression through the brain. A company called Neuromatic Devices in Atlanta, Georgia, offers machines based on
Boyden and Forest's technology, but did not disclose pricing or sales figures to Nature.

At the University of Texas at Austin, researchers have created a similar auto-patching system that is controlled in the MATLAB computing environment. This system uses a slightly different algorithm to decide when to start suctioning, and it succeeds in patch-clamping cells in mice about $17 \%$ of the time ${ }^{3}$. Neuroscientist Niraj Desai, who led the team, says that he hopes to incorporate more-sophisticated algorithms.

Some researchers question whether the recording robots will ever surpass the best human experts. "The elements that go into the human's decisions may be richer than can be captured by the machine," says neuroscientist Michael Hausser at University College London. But he adds that the technologies could still be a huge boon to novices. Others suggest that the robots could help users of all skill levels in lengthy or complex experiments, in which human fatigue becomes a limiting factor.

At the Allen Institute for Brain Science in Seattle, Washington, researchers have developed an automated system to assist in the even more challenging 'image-guided' variant of the technique. In this version, instead of blindly bumping into neurons with a pipette, scientists target specific neurons near the brain's surface using a two-photon microscope. The procedure requires more coordination than blind patch-clamping because the scientist must constantly focus the microscope in addition to guiding the pipette and adjusting its internal pressure. "This is a technique that ideally would benefit from having three hands," says Hausser, an expert in image-guided patching.

The automated system constructs $3 \mathrm{D}$ images of the brain region of interest and allows users to digitally select the neuron that they want to record. Then, with the coordinates locked in, the device navigates the pipette into place. For now, researchers still need to patch onto the cell by hand, but Allen Institute neuroscientist and joint team leader $\mathrm{Lu} \mathrm{Li}$ says that eventually they hope to fully automate the procedure.

Whether these automation systems will be taken up widely by the neuroscience community remains to be seen. Each of the teams has made their code freely available for people to download: Boyden's group at autopatcher.org; Desai's team at clm.utexas.edu/robotpatch; and Li's team at the GitHub repository (go.nature. $\mathrm{com} /$ sgjpab). "Our hope is that we can help as many people as possible to answer questions about how neurons compute," Boyden says.

1. Kodandaramaiah, S. B. et al. Nature Protoc. 11, 634-654 (2016).

2. Kodandaramaiah, S. B. et al. Nature Methods 9 , 585-587 (2012).

3. Desai, N. S., Siegel, J. J., Taylor, W., Chitwood, R. A. \& Johnston, D. J. Neurophysiol. 114, 1331-1345 (2015).

\title{
Web widget nudges scientists to share their data
}

\section{Open Data Button launched to encourage public sharing of data sets.}

\section{BY DALMEET SINGH CHAWLA}

A free, web-based tool that promises to help its users to ask authors of research papers to share their data publiclyand to make such requests publicly trackable - launched in beta version on 7 March. The Open Data Button (opendatabutton.org), a downloadable web-browser extension, can be clicked when a reader is looking at a research paper and wants to see its underlying data, says Joseph McArthur, who is co-leading the project and is assistant director of the policy advocacy group The Right to Research Coalition (R2RC) in London.

When clicked, the button generates a template e-mail that the user can send to the paper's authors. It asks them to share the data supporting the paper, explains how to do so and - if the user has typed in the information - states why the data would be useful. All requests are simultaneously posted on the Open Data Button website, where anyone can comment on existing entries to note that they want access to the same data sets.

If the author replies - either with a web link or attached data file - "we ask our users to affirm that this is the data they wanted", says McArthur. The tool will chase authors once a week for four weeks after a request is filed, he adds, after which entries will be marked as 'failed'.

The project is mostly funded by a US $\$ 25,000$ grant from the non-profit Center for Open Science in Charlottesville, Virginia; the centre has promised to host any data files sent in reply on its own Open Science Framework repository. The aim is to encourage open data sharing, which is still far from the norm in research, McArthur notes, even though many journals are now asking authors to publish their data alongside their research papers.

"It is good to draw attention to an important problem such as data availability with a oneclick gadget," says Bernd Pulverer, chief editor of The EMBO Journal in Heidelberg, Germany. But a button by itself "is probably not quite going to cause the revolution", he says. There is still a reluctance - especially among biologists - to share data openly, because of fears of being scooped by competition, and because of the extra work required to make data sets open, he notes. Ultimately, McArthur hopes that the button will not be needed as it becomes the norm to share data openly. But progress could be slow - so he thinks that the tool can expect a long and useful life. 